\title{
Avaliação da automedicação entre estudantes de medicina de uma instituição de ensino de Alagoas
}

\author{
Evaluation of automedication between medical students in a teaching \\ institution from Alagoas
}

\section{Camila Suica do Nascimento ${ }^{1}$, Karla Morgana Mota de Araújoํㅜ, Daniela Britto Marinho de Gusmão ${ }^{1}$, Paula Moura Souza ${ }^{1}$, José Alfredo dos Santos Júnior ${ }^{2}$}

\begin{abstract}
Nascimento CS, Araújo KMM, Gusmão DBM, Souza PM, Santos Júnior JA. Avaliação da automedicação entre estudantes de medicina de uma instituição de ensino de Alagoas / Evaluation of automedication between medical students in a teaching institution from Alagoas. Rev Med (São Paulo). 2019 nov.-dez.;98(6):367-373.
\end{abstract}

RESUMO: O uso medicamentoso de substâncias químicas na sociedade moderna tem crescido gradativamente. Nesse contexto, a prática de automedicação por profissionais e acadêmicos da área de saúde tem sido cada vez mais frequente, levando a uma preocupação quando se avalia o consumo abusivo de psicofármacos. A partir dessa problemática, relevante à saúde pública, se faz importante desenvolver meios e métodos que possam orientar com cautela sobre os riscos do uso indiscriminado de medicamentos. Objetivo: Avaliar a prática de automedicação e as particularidades atribuídas a ela entre estudantes de medicina de uma instituição de ensino superior. Metodologia: trata-se de um estudo descritivo do tipo transversal com abordagem quantitativa realizado no Centro Universitário CESMAC através de questionários aplicados aos acadêmicos de medicina. Das 9 turmas disponíveis para realização da pesquisa, todas tiveram os dados coletados e seus resultados interpretados a partir de bancos de dados criados utilizando o programa Microsoft Office Excel. Resultados e Discussões: 280 voluntários relataram fazer uso de automedicação, sendo essa representada em sua maior parte pelos anti-inflamatórios e apenas 4 pessoas alegaram se utilizar o metilfenidato por conta própria antes das provas. Conclusão: É evidente que o hábito de automedicação de maneira inadequada acarreta em consequências indesejáveis e no mascaramento de doenças evolutivas, o que se configura, portanto, em uma atividade a ser prevenida, assim como a utilização racional de medicamentos e a recomendação de profissional especializado devem ser enfatizadas.

Descritores: Automedicação; Efeitos colaterais e reações adversas relacionados a medicamentos; Estudantes de medicina; Psicotrópicos/efeitos adversos; Brasil.

\begin{abstract}
The drug use of chemicals in modern society has gradually grown. In this context, the practice of self-medication by professionals and academics in the health field has been increasingly frequent, leading to a concern when evaluating abusive use of psychoactive drugs. From this problematic, relevant to Public Health, it is important to develop ways and methods that can guide carefully the indiscriminate use of medicines. Objective: Evaluate the practice of self-medication and the particularities attributed to it among medical students of a higher education institution. This is a cross-sectional descriptive study with a quantitative approach carried out at CESMAC University Center through questionnaires applied to medical students. Of the 9 groups available to perform the research, all had the data collected and their results interpreted from databases created using the Microsoft Office Excel program. Results: 280 volunteers reported using self-medication, which was mostly anti-inflammatory and only 4 people reported using methylphenidate on their own before the tests. Conclusion: It is evident that the habit of self-medication improperly entails undesirable consequences and in masking of evolutionary diseases, which is, therefore, an activity to be prevented, as well as the rational use of medicines and the recommendation of a specialized professional should be emphasized.
\end{abstract}

Keywords: Self medication; Drug-related side effects and adverse reactions; Students, medical; Psychotropic drugs/adverse effects; Brazil.

Agência de fomento: Conselho Nacional de Desenvolvimento Científico e Tecnológico (CNPq).

1. Acadêmica de medicina do Centro Universitário CESMAC, Maceió, AL. ORCID: Nascimento CS- https://orcid.org/0000-00031640-9874; Araújo KMM - https://orcid.org/0000-0003-1386-8848; Gusmão DBM - https://orcid.org/0000-0003-1446-4722; Souza PM - https://orcid.org/0000-0002-5421-5002. Email: camilasuica@hotmail.com, karlamorgana18@gmail.com,dani_gusmao5@ hotmail.com, paula_m_souza@hotmail.com.

2. Professor titular do curso de Biologia do Centro Universitário CESMAC. ORCID: https://orcid.org/0000-0002-1324-1861. E-mail: ajrsantus@hotmail.com.

Endereço para correspondência: Camila Suica do Nascimento. Rua Cônego Machado, 918, Farol. Maceió, AL. CEP: 57051-160.

E-mail: camilasuica@hotmail.com 


\section{INTRODUÇÃO}

A automedicação é definida como uma forma de autoatenção à saúde, que tem por objetivo trazer benefícios para o tratamento de doenças ou alívio de sintomas ${ }^{1}$. Essa prática é justificada, entre outros motivos, pelo difícil acesso da população à consulta médica, limitação do poder prescritivo relacionado a poucos profissionais de saúde e falhas na regulamentação e na fiscalização daqueles que administram o medicamento. Estudos ressaltam que para a realização dessa prática, muitos indivíduos fazem uso de receitas antigas, mesmo que estas não tenham sido prescritas para uso contínuo ${ }^{2,3}$.

A prática de automedicação por profissionais e acadêmicos da área de saúde tem sido cada vez mais frequente, levantando uma preocupação quando se avalia o consumo abusivo de psicofármacos. Segundo o Escritório das Nações Unidas sobre Drogas e Crime (UNODC) ${ }^{4}$, os derivados anfetamínicos estão entre as substâncias mais populares em todo o mundo, sendo o Brasil responsável pela maior parte das apreensões de metilenodioximetanfetamina (ecstasy) e metanfetamina que, mesmo em proporções baixas, está entre os estimulantes do sistema nervoso central (SNC) mais utilizados.

Outra substância estimulante bastante consumida sem indicação profissional é a cafeína, que faz parte do grupo das metilxantinas e é muito utilizada como suplemento desportivo e em dietas de emagrecimento devido a sua propriedade termogênica. Sua utilização em doses moderadas resulta em uma ativação cortical suave com aumento do nível de alerta e redução da fadiga. Porém, em doses muito altas, produz estimulação bulbar e convulsões potencialmente letais ${ }^{5,6}$.

Essas atividades excitatórias denotam a popularidade dos derivados de anfetamina e de cafeína entre universitários e profissionais, sendo o consumo dessas substâncias extremamente intensificado em consequência do calendário letivo. Alguns estudos explicitam relatos de acadêmicos deixando clara a relação do uso dessas substâncias com as atividades avaliativas ${ }^{7}$. Em janeiro de 2017 foi divulgada em mídia nacional o caso de dois estudantes que quase morreram após beber o equivalente a 300 xícaras de café. Os alunos participavam de um experimento de esportes da Universidade Northumbria, no Reino Unido, onde estudam. Eles deveriam receber 0,3 g da substância, mas ao invés disso receberam 100 vezes mais, ou seja, $30 \mathrm{~g}$, caracterizando uma overdose de cafeína, sendo necessária internação em Unidade de Terapia Intensiva (UTI) devido aos efeitos provocados pelo excesso desse estimulante ${ }^{8}$.

Tem-se discutido também o uso crescente e banal, sem qualquer indicação terapêutica, do metilfenidato (droga derivada da anfetamina utilizada no Transtorno do Déficit de Atenção e Hiperatividade - TDAH $)^{5}$, por universitários saudáveis que buscam apenas melhorar o desempenho em suas atividades acadêmicas e rendimento intelectual devido a suas propriedades psicoestimulantes ${ }^{7,9}$.

É evidente que o hábito de automedicação de maneira inadequada acarreta consequências indesejáveis e mascaramento de doenças evolutivas, o que se configura, portanto, em uma atividade a ser prevenida. A utilização racional de medicamentos e a recomendação de profissional especializado devem ser enfatizadas ${ }^{2}$. A partir dessa problemática, relevante à saúde pública, se faz importante desenvolver meios e métodos que possam orientar com cautela sobre os riscos do uso indiscriminado de medicamentos ${ }^{10}$. O presente estudo visa avaliar a prática de automedicação entre estudantes de medicina de uma instituição de ensino superior, objetivando verificar quais as classes medicamentosas e fármacos mais utilizados na automedicação pelos estudantes, observar o uso de psicoestimulantes naturais ou sintéticos pelos estudantes, com enfoque para o metilfenidato, relatar os principais motivos para a automedicação pelos discentes, analisar se há um aumento da automedicação no decorrer do curso, comparando o uso de medicamentos entre alunos calouros e veteranos e correlacionar a prática de automedicação com o gênero, idade e renda familiar do estudante.

\section{METODOLOGIA}

Trata-se de um estudo descritivo do tipo transversal com abordagem quantitativa realizado no Centro Universitário CESMAC. Para a elaboração do referencial teórico, foram selecionados artigos de revistas indexadas nas bases de dados Lilacs, SciELO e Pubmed, entre os meses de julho de 2006 a junho de 2018, que apresentaram pesquisas com perguntas semelhantes àquelas que constituem o questionário que integra este estudo.

Baseado no número de pessoas matriculadas em medicina fornecido pela coordenação de curso, que correspondia a 478 discentes, foi aplicado cálculo estatístico e obtida uma amostra de 214 estudantes, sendo este total dividido pelos sete períodos do curso em vigência no momento, obtendo uma amostra de 31 estudantes por cada período (primeiro ao sétimo períodos) ${ }^{11}$.

A amostra final foi composta por acadêmicos do curso de medicina regularmente matriculados e que preencheram corretamente o termo de consentimento livre e esclarecido (TCLE), excluindo-se aqueles estudantes que não estavam presentes em sala de aula durante a distribuição dos questionários e aqueles que se recusaram a respondê-lo.

Inicialmente foi realizado um contato prévio com a coordenação do curso para combinar o melhor dia e horário em que se poderia utilizar 15 minutos em sala de aula para explicitar os objetivos, procedimentos, riscos e benefícios da pesquisa e, em seguida, proceder ao convite verbal à participação do estudo.

Aos indivíduos que aceitaram participar da pesquisa, foi entregue um envelope pardo, sem identificação, 
contendo duas vias do TCLE, informando-lhes que deveria ser devolvida apenas uma via do TCLE, assinada a última folha e rubricada as demais. Foram disponibilizadas as opções de proceder à leitura e assinatura, com devolução do TCLE no mesmo dia do recrutamento ou levar para fazer esses procedimentos em sua residência para devolução posterior. Os pesquisadores retornaram às salas de aula na semana posterior para recolher os envelopes com os TCLE. A coleta dos envelopes com uma via devidamente assinada do TCLE foi feita por meio de uma urna.

A coleta dos dados foi realizada através de questionário, contendo variáveis independentes como idade, gênero e renda familiar e variáveis dependentes como o uso, motivos, dependência e frequência da automedicação; conhecimento sobre o que é automedicação; conhecimento sobre os riscos, contraindicações e efeitos colaterais da automedicação; casos de intoxicação por automedicação; ato de recomendar medicamentos a familiares e amigos; uso de medicamentos antes das provas; principais medicamentos utilizados por automedicação; uso de estimulantes para ficar acordado; mudança da prática de automedicação no decorrer do curso; uso de medicamento com e sem prescrição; hábito de ler a bula; perguntas específicas sobre a automedicação com metilfenidato (Ritalina ${ }^{\circledR}$ ).

O questionário foi entregue dentro de um envelope pardo, não identificado, no mesmo dia, local e momento do recrutamento e aquisição do TCLE, para ser respondido a punho pelo indivíduo. O participante teve a opção de responder no mesmo dia ou levar para casa e devolver dentro de um prazo de uma semana.

\section{RESULTADOS}

Após a finalização da coleta de dados, deu-se início à digitação e tabulação dos mesmos em planilhas do programa Microsoft Excel, sendo posteriormente submetidos à apuração e interpretação dos resultados.

A amostra final foi composta por 284 voluntários, dos quais 188 identificaram-se com o gênero feminino, 88 com o masculino e 8 preferiram não informar, atingindo uma idade média de 22,9 anos (faixa etária entre 18 e 41 anos). Devido à baixa adesão dos voluntários à resposta da pergunta sobre a renda familiar não foi possível obter resultados que refletissem a realidade socioeconômica das turmas.

De início, os voluntários da pesquisa foram questionados sobre o que eles entendiam por automedicação: 241 participantes afirmaram que a automedicação consiste no ato de medicar-se por conta própria, sem prescrição médica. As demais respostas dissertavam sobre o uso de medicação sem prescrição de "profissional habilitado", incluindo no poder prescritivo não só médicos, como também dentistas, farmacêuticos e profissionais de saúde de maneira geral.

Da totalidade de participantes da pesquisa, apenas $1,4 \%$ (4/284) relataram não fazer uso de automedicação, como evidenciado no Quadro 1. Porém, dentre aqueles que confirmaram a prática deste ato, a principal justificativa para tal foi a praticidade e a comodidade que a automedicação traz, sendo este motivo relatado por $97,1 \%(272 / 280)$ das pessoas, seguido pela facilidade na compra dos medicamentos em 72,5\% dos casos (203/280). Menos prevalentes foram a falta de acesso aos serviços de saúde, com 9,3\% (26/280), a falta de dinheiro com 4,6\% (13/280), e outros que somaram $6,1 \%(17 / 280)$ como: falta de tempo de ir ao médico (5), início rápido do tratamento (1), ser profissional odontólogo (1), demora no atendimento (3), conhecimento prévio do efeito farmacológico da droga (5), subestimar a gravidade da doença (1) e "questões culturais" (1).

Quadro 1 - Descrição das questões objetivas

\begin{tabular}{|c|c|c|}
\hline Questões & Sim & Não \\
\hline Fez uso de medicação sem prescrição médica? & 280 & 4 \\
\hline Aconselhou-se com terceiros acerca de alguma medicação? & 220 & 64 \\
\hline Você acredita que a automedicação poderia trazer algum dano à saúde? & 278 & 6 \\
\hline Houve algum episódio diagnosticado de intoxicação? & 7 & 275 \\
\hline Você considera que é dependente dessa automedicação? & 20 & 262 \\
\hline $\begin{array}{l}\text { Você tem conhecimento das contraindicações e dos efeitos colaterais dos medicamentos automedicados por } \\
\text { você? }\end{array}$ & 227 & 61 \\
\hline Você recomendaria medicamentos aos familiares e amigos? & 205 & 74 \\
\hline Você sabe onde pode comprar Ritalina ${ }^{\circledR}$ sem receita médica? & 42 & 239 \\
\hline Já usou algum tipo de anfetamina (exceto Ritalina $\left.{ }^{\circledR}\right)$ ? & 26 & 256 \\
\hline Na sua opinião, a Ritalina ${ }^{\circledR}$ é uma droga utilizada de forma frequente pelos universitários do seu curso? & 187 & 95 \\
\hline Na sua opinião, a Ritalina ${ }^{\circledR}$ é uma droga utilizada de forma frequente pelos universitários & 88 & 186 \\
\hline
\end{tabular}

Fonte: dados da pesquisa. 
Quando questionados sobre a leitura das bulas antes da administração de medicamentos, 31,8\% (89/280) responderam que sempre leem a bula, 44,3\% (124/280) afirmaram que leem quando possível e $24 \%$ (67/280) que não leem a bula em nenhuma situação. Já em relação à frequência da prática nos últimos 12 meses, 48,2\% $(135 / 280)$ das pessoas responderam que o fizeram entre 1 a 5 vezes, $23,2 \%(65 / 280)$ que a prática foi entre 6 a 10 vezes, 16,4\% (46/280) incontáveis vezes, 11,8\% (33/280) mais que 10 vezes e apenas 1 pessoa não respondeu a essa pergunta.
O Gráfico 1 evidencia as classes de medicamentos mais utilizadas sem orientação médica, com destaque para os anti-inflamatórios não-esteroidais (AINE), sendo a classe com maior uso, seguidos pelos antialérgicos, descongestionantes nasais, anti-inflamatórios esteroidais (AIE), antibióticos, analgésicos e antidepressivos. Outras classes foram citadas em menores quantidades: anticoncepcionais (7), medicamentos para sintomas gastrointestinais (5), relaxantes musculares (2), antifúngicos (2), neurolépticos (2), psicoestimulantes (1), antiparasitários (1), ansiolíticos (1) e antitérmicos (1).

Gráfico 1 - Classes de medicamentos mais utilizadas na automedicação

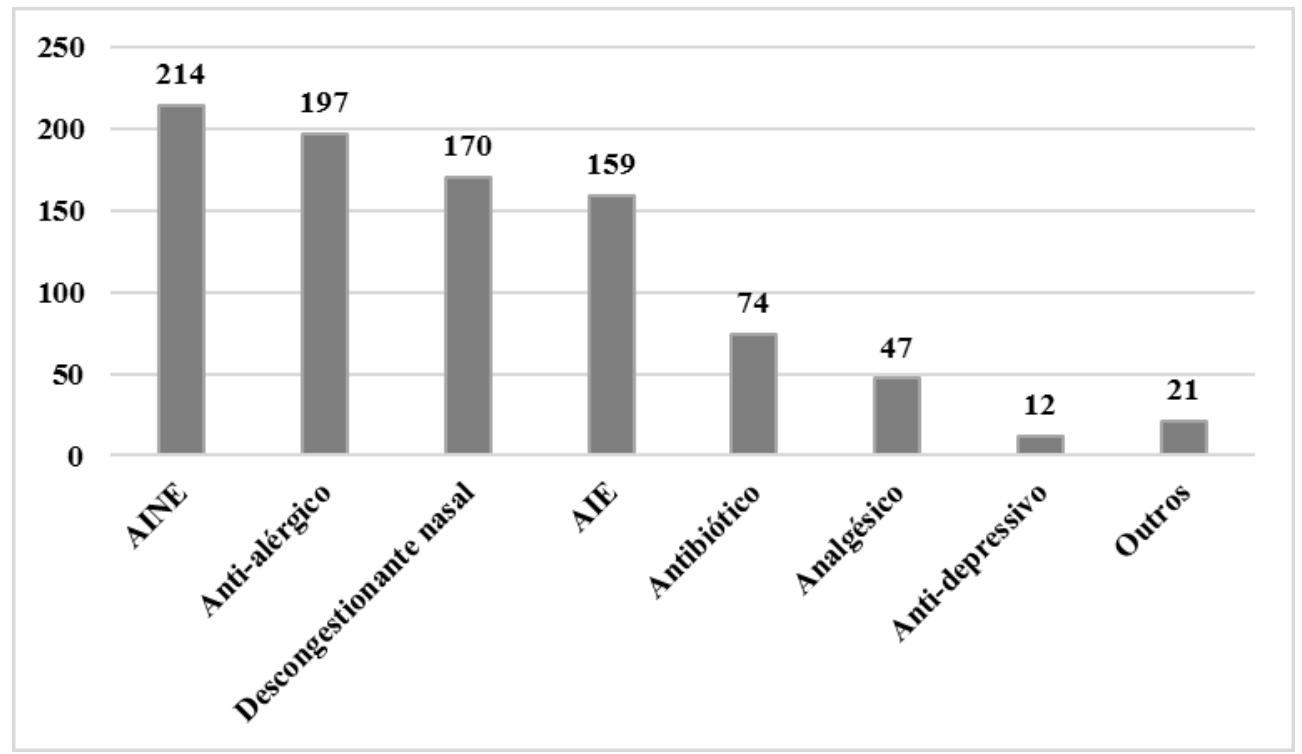

Fonte: dados da pesquisa.

De maneira semelhante, as classes que mais são compradas sem receita também foram os anti-inflamatórios (AINE e AIE), seguidos dos antialérgicos, logo depois vieram os analgésicos e os descongestionantes nasais. Todas essas classes de medicamentos podem ser vendidas isentas de prescrição, segundo lista elaborada pela Anvisa e publicada no Diário Oficial da União em 30 de setembro de $2016^{13}$. Entre as classes que necessitam de prescrição, foram adquiridas sem a mesma: antibióticos (35), antidepressivos (2), sedativo (1), metilfenidato (1), imunossupressor (1) e isotretinoína (1).

Quando arguidos a respeito de quais sintomas os fizeram recorrer à automedicação, os mais prevalentes foram cefaleia em $90 \%$ dos casos (252/280), gripe/resfriado em 78,6\% (220/280), febre em 75,7\% (212/280), outra dor em 63,9\% (179/280), tosse em 53,2\% (149/280), infecções em 31,8\% (89/280) e, por fim, prevenção em $14,3 \%(40 / 280)$.

No tocante à utilização de estimulantes para estudar ou se manter acordado, sem levar em consideração que o estudante tenha ou não prescrição para o uso dessas substâncias, 26,4\% (74/280) informaram fazer uso de drogas para essa finalidade, sendo as classes mais mencionadas cafeína (59), energéticos (9), metilfenidato (6), guaraná (5), modafinila (2), termogênico (2), bupropiona (1), taurina (1) e floral (1).

Sobre quais as principais influências que os levaram a realizar a automedicação, a resposta mais prevalente foi o conhecimento próprio do indivíduo em $67,1 \%$ casos (188/280) e a opinião de familiares, vizinhos e amigos, que somaram 59,3\% (166/280); outros 42,8\% (120/280) alegaram fazer uso de prescrição antiga para adquirir novos medicamentos e, em menor quantidade, a intervenção de farmacêuticos/funcionários da farmácia representou 13,2\% (37/280) e de propagandas 8,2\% (23/280).

Em relação ao hábito da automedicação após o estudante ingressar no curso de medicina e no decorrer deste, $60,4 \%$ (169/280) referiram que a prática se manteve igual a antes de iniciar a faculdade, enquanto $28,2 \%$ $(79 / 280)$ afirmaram que houve um aumento e apenas 10,0\% (28/280) relataram uma diminuição; 4 estudantes se abstiveram da resposta. 
No Gráfico 2, é possível evidenciar uma relação entre o número de pessoas que alegaram aumento da automedicação, com o avançar dos semestres cursados, de maneira que no primeiro período ( $\mathrm{A}$ e $\mathrm{B}$ ) esse número foi de 10 pessoas, enquanto no sétimo período (A e B) esse número aumentou para 22 pessoas. De acordo com a grade curricular do Curso de Medicina do Centro Universitário CESMAC, a disciplina de farmacologia é ministrada durante o terceiro e o quarto períodos, o que pode justificar um aumento dessa automedicação baseada em conhecimentos próprios principalmente após o quarto período do curso.

Gráfico 2 - Automedicação ao longo do curso

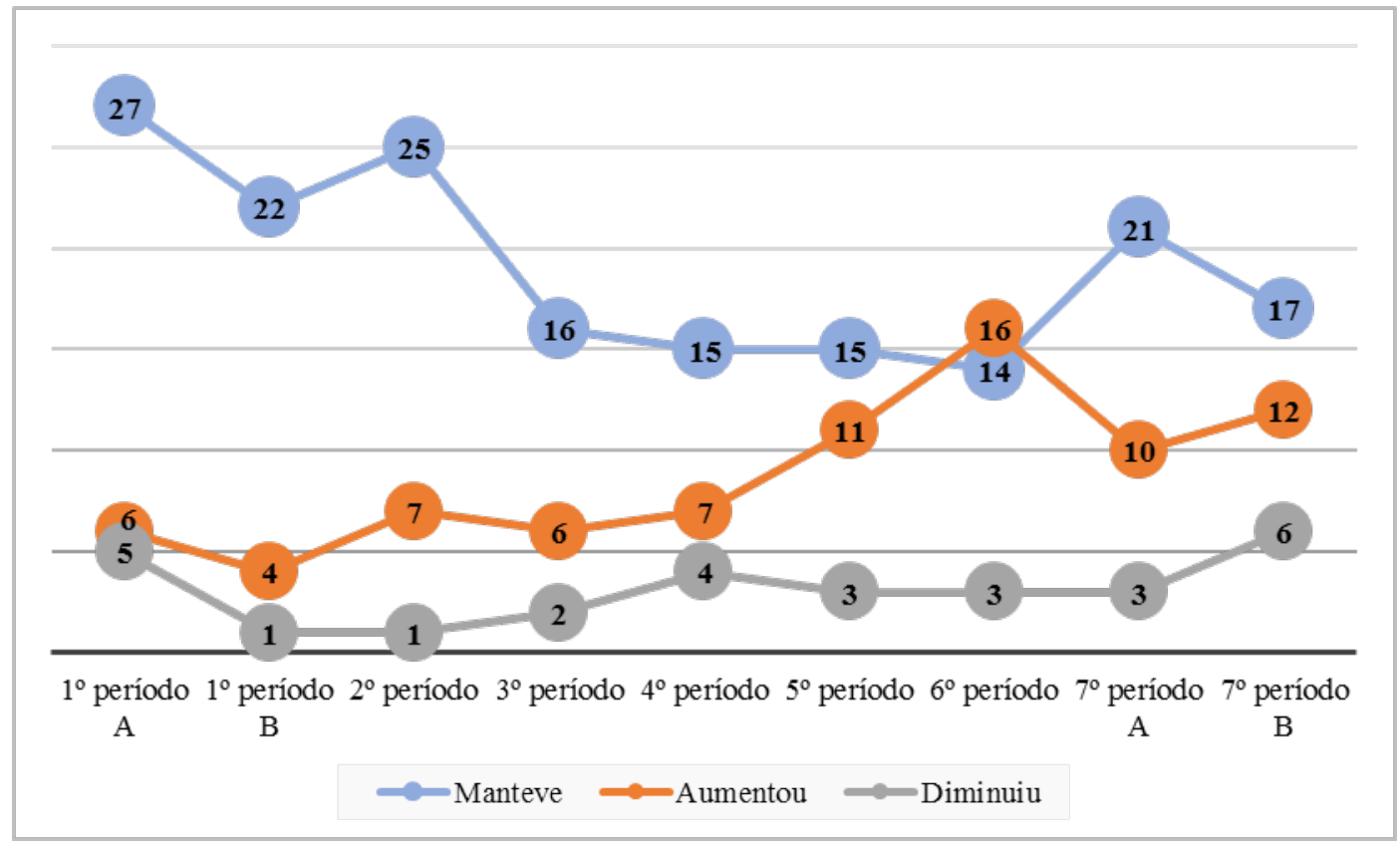

Fonte: dados da pesquisa.

\section{DISCUSSÃO}

Este trabalho foi realizado no município de Maceió/AL e apresenta dados que confirmam o alto índice da prática da automedicação entre os estudantes de medicina do Centro Universitário CESMAC, alcançando uma prevalência de 98,6\% (280/284), um resultado significativamente superior quando comparado a valores nacionais relatados em pesquisas anteriores feitas na Universidade de Ribeirão Preto (UNAERP), que apresentou índice de automedicação de 92\%, Universidade Federal de Juiz de Fora (UFJF), com 76\%, Universidade Católica de Pelotas (UCPel), com 85,7\%, e Universidade Federal de Pelotas (UFPel), com 94\%. Pesquisa semelhante mostrou que a prevalência da automedicação é de 49,3\% entre os estudantes de medicina de uma universidade pública na cidade de Dammam, Arábia Saudita ${ }^{13-16}$.

Ao considerar apenas os alunos do primeiro período de medicina do CESMAC, 98,4\% (60/61) declararam se automedicar, o que pode ser atribuído a hábitos préexistentes em sua rotina. Já entre os acadêmicos do último período vigente na época em que o estudo foi realizado $\left(7^{\circ}\right.$ período), a prática foi relatada por $100 \%(69 / 69)$. Esses resultados indicam que a automedicação está presente em todos os períodos do curso, sofrendo leve aumento à medida que o curso avança e guardando pouca relação com o conhecimento adquirido ao longo da formação médica. O estudo realizado na UNAERP demonstrou que, entre os alunos ingressantes no curso, a prática da automedicação foi relatada por $86,6 \%$, também sendo atribuída a hábitos já existentes entre os acadêmicos.

Além disso, a maior parte dos acadêmicos interrogados, 72,2\% (205/284), informou já ter indicado medicamentos para familiares e amigos, prática considerada ilegal pelo Código de Ética Médica. O mesmo ocorreu entre os estudantes da UNAERP, onde essa porcentagem correspondeu a $69,5 \%{ }^{14,17}$.

Segundo Fallah et al. ${ }^{18}$, os estudantes de medicina estão mais propensos ao uso indiscriminado e sem indicação terapêutica de drogas estimulantes como as anfetaminas (principalmente o metilfenidato), devido às exigências de suas condições acadêmicas, que incluem vigília prolongada, estresse, melhorias na concentração e no desempenho acadêmico. Essas ações são compatíveis com os efeitos esperados do metilfenidato, que, apesar de ainda não terem sido completamente elucidados, acredita-se 
atuar inibindo a recaptação de dopamina e noradrenalina, responsáveis pelo aumento da pressão arterial e os níveis de glicose no sangue, aumento da sensação de euforia e bem-estar, aumento do estado de vigília, melhoraria na memória, concentração e aprendizagem, contribuindo para sua popularidade entre os acadêmicos ${ }^{19}$.

Um estudo realizado na Universidade de Ciências Médicas de Babol, no Irã, durante os anos de 2014 e 2015, que contou com 444 voluntários alunos do curso de medicina, demonstrou que $11 \%$ dos indivíduos alegaram fazer uso de estimulantes e, dentre eles, 6,5\% especificaram o metilfenidato como droga de escolha, enquanto $2,5 \%$ fizeram uso de outras anfetaminas ${ }^{18}$. Por sua vez, no Centro Universitário CESMAC, 26,4\% (74/280) dos acadêmicos de medicina confirmaram fazer uso de estimulantes, sendo o metilfenidato relatado por $2,1 \%(6 / 280)$ dos estudantes e outras anfetaminas por $9,3 \%(26 / 280)$, como observado no Quadro 1. Dessa maneira, constatou-se que o uso de estimulantes, como o metilfenidato e outras anfetaminas foi mais prevalente no Centro Universitário CESMAC quando comparado com a Universidade de Ciências Médicas de Babol.

De acordo com Aquino et al. $^{2}$, os analgésicos e os anti-inflamatórios são as classes de medicamentos mais populares no Brasil, fato este que condiz com os resultados de estudos feitos com acadêmicos de medicina na Universidade Metropolitana de Santos (UNIMES), na UNAERP e na Faculdade de Medicina de Marília (FAMEMA). A automedicação feita com analgésicos foi relatada por $45,4 \%$ dos estudantes na Unimes, 90,2\% na UNAERP e 94,2\% na Famema, já os AINE foram utilizados indiscriminadamente por $15,7 \%$ dos acadêmicos de medicina da Unimes, $85,3 \%$ da UNAERP e $85,1 \%$ da Famema ${ }^{3,14,20}$. No CESMAC, os resultados mostraram-se um pouco diferentes, uma vez que a classe mais utilizada nesta instituição foram os AINE com 76,4\% (214/280) e, em segundo lugar, vieram os antialérgicos com 70,3\% (197/280). Os analgésicos chegaram apenas à quinta posição entre os mais utilizados, com 16,8\% (47/280) de popularidade entre os estudantes.

No estudo realizado entre acadêmicos de medicina da UFJF, a principal motivação para a prática da automedicação foi julgar que não seria necessário ir ao médico para tratar o sintoma em questão, motivo relatado por $90,36 \%$ dos estudantes, seguido por apenas $3,61 \%$ que alegou dificuldade de acesso ao médico ${ }^{13}$. Em contrapartida, no levantamento realizado no CESMAC, a principal justificativa para tal foi a praticidade e a comodidade que a automedicação traz, segundo 97,1\% (272/280) das pessoas, enquanto a dificuldade de acesso aos serviços de saúde foi relatada por apenas 9,3\% (26/280), dos participantes.

No tocante às principais influências que levaram à automedicação, entre os estudantes da UFJF foi observado que conselhos de amigos e parentes foi o motivo prevaleceu, sendo relatado por $23,4 \%$ das pessoas. Porém, no CESMAC, este mesmo motivo foi apenas o segundo mais relevante, sendo referido por 59,3\% (166/280) dos alunos, e, em primeiro lugar, veio o conhecimento próprio do indivíduo, alternativa escolhida por 67,1\% (188/280) dos voluntários da pesquisa, de maneira semelhante ao resultado obtido na FAMEMA, onde $83,4 \%$ dos voluntários alegaram este como o motivo mais relevante para a automedicação. Tanto no CESMAC, como na FAMEMA e na UNIMES, o sintoma que mais motivou a automedicação foi a cefaleia, com prevalência de 90,0\% (252/280), 85,4\% e $14,6 \%$ respectivamente ${ }^{3,13,14,20}$.

Quando questionados sobre a leitura das bulas antes da automedicação, 31,8\% (89/280) dos estudantes do CESMAC responderam que sempre leem a bula e 44,3\% (124/280) afirmaram que leem quando possível, representando um total próximo aos $86,52 \%$ que confirmaram a leitura das bulas na UFJF e aos $78,7 \%$ da FAMEMA $^{13,20}$.

A automedicação entre acadêmicos de medicina tem se tornado uma importante problemática atual, de maneira que a necessidade de novos estudos, sob pontos de vista distintos, é evidente para uma melhor elucidação a cerca de novas abordagens do problema. A amostra da pesquisa foi composta por alunos de apenas uma instituição de ensino, o que pode deixá-los sob a influência dos mesmos fatores em relação à prática de automedicação, além de que, para a realização do estudo, os pesquisadores dependiam da adesão voluntária dos participantes à pesquisa. Apesar disso, os dados obtidos representam o único estudo disponível sobre a automedicação entre acadêmicos de medicina de Alagoas, fornecendo dados importantes não só para este estado, mas que são necessários também à fomentação das pesquisas sobre o assunto a nível nacional.

\section{CONCLUSÃO}

A prevalência de automedicação em acadêmicos de medicina do CESMAC é significativamente superior aos descritos em estudos semelhantes realizados anteriormente no Brasil e em outros países. Esses resultados denotam a necessidade de intervenção das estruturas educacionais com a finalidade de alertar cientificamente sobre os riscos desta prática no meio acadêmico. Ademais, questões éticas e psicológicas merecem especial atenção dos responsáveis pela formação médica, visto que a problemática tangencia a formação de futuros profissionais da saúde que precisarão agir de maneira consciente com relação ao tema ao longo de toda a vida profissional, principalmente pelo fato desta classe ser formadora de opinião quanto ao uso racional de medicamentos. 
Contribuição dos autores: Nascimento CS e Araújo KMM: elaboração do projeto inicial que foi submetido à Plataforma Brasil e ao Comitê de Ética e Pesquisa do Centro Universitário CESMAC. Aplicação e coleta do TCLE e dos questionários. Tabulação dos dados. Análise dos dados. Pesquisa do referencial teórico em bases de dados. Escrita do artigo contendo os dados coletados e devidamente analisados. Adequação do artigo às normas da Revista de Medicina, incluindo traduções, quando necessário, com posterior submissão à plataforma para publicação. Correção do texto do artigo segundo orientações dos avaliadores da Revista de Medicina. Gusmão DBM e Souza PM: Aplicação e coleta do TCLE e dos questionários. Tabulação dos dados. Júnior JAS: orientações acerca da elaboração do projeto inicial e desenvolvimento do TCLE e do questionário. Aplicação de métodos estatísticos para definição de amostra inicial da pesquisa.

\section{REFERÊNCIAS}

1. Damasceno DD, Terra FS, Zanetti HHV, D'Andréa EV, Silva HLR, Leite JA. Automedicação entre graduandos de enfermagem, farmácia e odontologia da Universidade Federal de Alfenas. REME Rev Min Enf. 2007;11(1):4852. Disponível em: https://www.academia.edu/30257949/ Automedica\%C3\%A7\%C3\%A3o_entre_graduandos_ de_enfermagem_farm $\% \mathrm{C} 3 \% \mathrm{~A} 1 \mathrm{cia}$ _e_odontologia_da_ Universidade_Federal_de_Alfenas.

2. Aquino DS, Barros JAC, Silva MDP. A automedicação e os acadêmicos da área de saúde. Rev Ciên Saúde Coletiva. 2010;15(5):2533-8. https://doi.org/10.1590/s141381232010000500027 .

3. Fonseca FIRM, Dedivitis RA, Smokou A, Lascane E, Cavalheiro RA, Ribeiro EF, et al. Frequência de automedicação entre acadêmicos de faculdade de medicina. Diagn Tratamento. 2010;15(2):53-7. Disponível em: http:// files.bvs.br/upload/S/1413-9979/2010/v15n2/a53-57.pdf.

4. United Nations Office on Drugs and Crime - UNODC. Amphetamines and ecstasy: 2011 Global ATS Assessment. Austria; 2011 [cited 2017 May 31]. Available from: https://www.unodc.org/documents/scientific/ATS_Global_ Assessment_2011.pdf.

5. Katzung BG, Trevor AJ. Farmacologia básica e clínica. 12a ed. Porto Alegre: Artmed; 2014.

6. Golan DE, Tashjian AH, Armstrong EJ, Armstrong AW. Princípios de farmacologia. 3a ed. Rio de Janeiro: Guanabara Koogan; 2009.

7. Tsuda CA, Christoff AO. Avaliação do padrão de uso de estimulantes em uma faculdade de Curitiba-PR. Cad Escola Saúde. 2015;1(13):116-32. https://doi. org/10.11606/d.22.2005.tde-16122005-112919.

8. Portal R7. Estudantes ficam à beira da morte por overdose de cafeína. Reino Unido. 2017 jan [citado 31 maio 2017]. Disponível em: http://noticias.r7.com/saude/estudantesficam-a-beira-da-morte-por-overdose-de-cafeina-26012017.

9. Cruz TCSC, Barreto Junior EPS, Gama MLM, Maia LCM, Melo Filho MJX, Manganotti Neto O, et al. Uso não-prescrito de metilfenidato entre estudantes de Medicina da Universidade Federal da Bahia. Gazeta Méd Bahia. 2011;81(1):3-6. https:// doi.org/10.5327/z2176-947820180352.
10. Moreau RLM, Siqueira MEPB. Ciências farmacêuticas: toxicologia analítica. Rio de Janeiro: Guanabara Koogan; 2014.

11. Vieira S. Bioestatística. Rio de Janeiro: Campus; 2003.

12. Brasil. Instrução Normativa n. 11, de 29 de setembro de 2016. Dispõe sobre a lista de medicamentos isentos de prescrição. 2016 set 1: 99 .

13. Chehuen Neto JA, Sirimarco MT, Choi CMK, Barreto AU, Souza JB. Automedicação entre Estudantes da Faculdade de Medicina da Universidade Federal de Juiz de Fora. Rev HU. 2006;32(3):59-64. https://doi.org/10.5327/z2447211520171600074

14. Silva RCG, Oliveira TM, Casimiro TS, Vieira KAM, Tardivo MT, Junior MF, et al. Automedicação em acadêmicos do curso de medicina. Medicina. 2012;45(1):5-11. https://doi. org/10.11606/issn.2176-7262.v45i1p5-11.

15. Pilger, M.C, Dombrowski, G, Rebelo, M, Tomasi, E. Automedicação entre acadêmicos de Medicina das Universidades Católica e Federal de Pelotas/RS. Rev AMRIGS. 2016;60(1):26-31.

16. Albusalih FA, Naqvi AA, Ahmad R, Ahmad N. Prevalence of Self-Medication among Students of Pharmacy and Medicine Colleges of a Public Sector University in Dammam City, Saudi Arabia. Pharmacy. 2017;5(4):51-63. https://doi. org/10.3390/pharmacy5030051

17. Conselho Federal de Medicina. Código de ética médica: resolução CFM n.1931/09. Brasília: CFM; 2010.

18. Fallah G, Moudi S, Hamidia A, Bijani A. Stimulant use in medical students and residents requires more careful attention. Caspian J Intern Med. 2018;9(1):87-91.

19. Ritalina ${ }^{\circledR}$ e Ritalina LA ${ }^{\circledR}$ [Bula]. São Paulo: Novartis Biociências SA; 2014.

20. Masson W, Furtado PL, Lazarini CA, Conterno LO. Automedicação entre acadêmicos do curso de Medicina da Faculdade de Medicina de Marília. Rev Bras Pesqui Saúde. 2012;14(4):82-9. https://doi.org/10.21722/rbps.v14i4.5123.

Recebido: 22.02 .19

Aceito: 27.09 .19 